\title{
COGNITION, DOULEUR, CONSCIENCE ET BIEN-ETRE DES ANIMAUX
}

\section{COGNITION, CONSCIOUSNESS, PAIN, AND ANIMAL WELL-BEING}

Par Bertrand L. DEPUTTE ${ }^{1}$ et Pierre LE NEINDRE²

(Communication présentée lors de la Séance du président de l'Académie Vétérinaire de France consacrée au bien-être animal le 5 Novembre 2020, Manuscrit accepté le 5 Mars 2021)

\begin{abstract}
RÉSUMÉ
Cet article, propose une synthèse des différents concepts-clés éthologiques qui constituent le cadre de l'analyse du bien-être animal. Le premier constat est que les animaux sur lesquels nous travaillons ne sont pas des machines réagissant à des schémas préétablis. Ils ont une liberté d'action et prennent des décisions qui font appel à des capacités cognitives leur permettant d'analyser les évènements qu'ils vivent. Liée à cette cognition, la conscience est la réflexivité de l'individu sur son état et ses actions. Les résultats scientifiques récents montrent des compétences chez l'animal telles qu'émotion, métacognition et mémoire épisodique. Ils permettent de caractériser attentes, plaisirs et douleurs. Ces qualités constituent la base permettant de définir le bien-être animal, c'est-à-dire l'état de satisfaction ou d'insatisfaction. Les conséquences pratiques de ces différents concepts sont explicitées en termes d'élevage et d'abattage.
\end{abstract}

Mots-clés : bien-être animal, éthologie, cognition, métacognition, conscience, douleur

\begin{abstract}
-ABSTRACT
The key ethological concepts important for analysing animal well-being are described. The first statement is that animals are not acting as machines following preestablished schemes. They have their own will and take decisions using their cognitive abilities for adapting to the situations. One key concept is their consciousness which is the means through which the animal reflects on its situation. Recent scientific results provided information that animal can have to some extent the same conscious abilities as a human, including emotions, metacognition and episodic memory. These abilities are essential for the animals to qualify their expectations, pleasures and pains. Evaluation of those abilities are the bases for qualifying animal welfare in terms of satisfaction or dissatisfaction. The practical consequences of those concepts are described especially for breeding.

Key words : animal well-being, ethology, cognition, metacognition, consciousness, pain
\end{abstract}

1. Professeur honoraire, Ecole Nationale Vétérinaire d'Alfort, 12 Chemin des châteaux 35380 Plélan le Grand, France. Courriel : bert.deputte@wanadoo.fr

2. Directeur de Recherches, INRA, 4 rue de l'avancée, 56290 Port-Louis, France. Courriel : pierre.le-neindre@orange.fr 


\section{INTRODUCTION}

Le concept de bien-être et son extension aux animaux est un terme de plus en plus employé par un public de plus en plus large. Chacun y met un sens correspondant à ses intérêts ; argument/ étiquette de marketing (thalasso, produit alimentaire, boisson, bricolage, radiateurs électriques, etc.) ou même revenu annuel équivalent à un certain pourcentage du PIB national (Inglehart \& Klingemann 2000). Cela conduit à un galvaudage de ce concept. Dans le Petit Robert (1977), le bien-être est défini comme une " Sensation agréable procurée par la satisfaction de besoins physiques, l'absence de tensions psychologiques - Euphorie, agrément, aise, béatitude, bonheur, félicité, jouissance, plaisir, quiétude, satisfaction, sérénité ». Pour les humains, le concept de bien-être est culturellement marqué. Appliqué aux animaux, le bien-être est souvent employé comme synonyme "d'amour ", de confort ou de soins. Milhaud (2007) suggère que le mot « welfare " soit traduit par "bien-être " lorsqu'il est question du ressenti de l'animal et par bientraitance lorsqu'il est question des actions de l'homme pour améliorer le bien-être des animaux.

Pour ce qui concerne le bien-être c'est bien l'animal qui est au centre de la définition. Il faut se placer du côté de ses compétences biologiques, de ses adaptations, de son histoire évolutive même lorsqu'elle a été infléchie par la sélection artificielle. Il faut comprendre les émotions des animaux, leur vie de relation, leurs réponses face aux contraintes quand il s'agit d'animaux sauvages confinés pour diverses raisons (zoos, expérimentation et recherche) et plus encore quand il s'agit d'animaux qu'ils soient de rente ou de compagnie. Il est donc question de se mettre du côté de l'animal pour comprendre ses émotions et ses compétences disponibles pour répondre à des contraintes nouvelles. Pour cela, il est nécessaire de pouvoir disposer d'une connaissance opérationnelle de ces compétences. De cette connaissance pourront découler des mesures visant à statuer si les animaux sont en situation de bien-être ou pas. Cette évaluation permettra de modifier et d'adapter les conditions de vie des individus pour éventuellement leur permettre de retrouver une sensation de bien-être si elles n'étaient pas optimales. Le bien-être a une dimension subjective donc individuelle. Mettre en place des mesures de bientraitance (Milhaud 2006) pour des cohortes ou des groupes d'individus est une nécessité mais ce n'est pas suffisant si l'on ne considère pas le comportement de chaque individu.

\section{HISTOIRE DU BIEN-ETRE ANIMAL}

Le bien-être animal implique l'analyse du comportement Dès 1858, un an avant que Darwin ne publie son livre fondateur de la théorie de l'évolution (1859), Claude Bernard écrivait : "Tout le monde croit être apte à interroger la nature par l'expérimentation : c'est là le plus souvent une grave erreur. Mais remarquons bien que cette espèce de confiance avec laquelle on aborde les questions les plus compliquées ne se rencontre guère que dans les choses du domaine de la médecine et de la physiologie. Lorsqu'une personne compétente traite un sujet spécial de chimie ou de physique, ceux qui ne sont ni physiciens ni chimistes, se taisent et évitent de se mêler à une discussion pour laquelle ils se sentent incompétents. Eh bien, la même chose n'a plus lieu quand il s'agit de médecine, tout le monde croit pouvoir en parler et en parle " (Bernard 1858, p.6-7). Cette réflexion s'applique parfaitement à l'éthologie qui est la biologie du comportement. Analyser le comportement des animaux relève de la méthode scientifique, mais parler du comportement des animaux n'appartient qu'au discours, propre à tout humain. Observer des animaux dans leur vie de relation et dans leurs activités quotidiennes appartient à tout un chacun. Mais l'établissement des connaissances scientifiques de ces comportements, et leur analyse, appartiennent aux chercheurs - éthologistes et psychologues comparatistes (cf. Morgan 1894). La question fondamentale de Tinbergen, l'un des fondateurs de l'éthologie est : "Why do these animals behave as they do, now ? " (Tinbergen 1963). Or c'est sur la base de ces connaissances scientifiques que le bien-être animal peut et doit être évalué.

Les prémisses : le bien-être animal comme finalité de la vie de relation

Le concept du bien-être animal relève de l'analyse du comportement et non du discours, surtout lorsqu'il s'agit de transcrire les réponses des animaux et non de parler à leur place. Il est intéressant de constater que Descartes (édition de 1996) avait tort en assimilant les animaux à des robots, et que, en revanche, Lamarck dans un autre registre avait raison lorsqu'il écrivait en 1815 à propos de 2 catégories d'animaux qu'il distinguait - les animaux sensibles et les animaux intelligents (Lamarck 1835, p 220-223) : "Chez les animaux sensibles il existe un penchant à la conservation de leur être, parce qu'ils possèdent un sentiment intérieur qui le produit et qui les fait agir lorsque des besoins le sollicitent. Or, comme tout besoin est un mal-être jusqu'à ce qu'il soit satisfait le penchant à la conservation les porte à fuir le mal-être, lorsqu'ils l'éprouvent. Ils possèdent un penchant : $1^{\circ} \mathrm{A}$ fuir la douleur, lorsqu'ils la ressentent ; $2^{\circ} \mathrm{A}$ chercher et saisir leur nourriture, lorsqu'ils en éprouvent le besoin ; $3^{\circ} \mathrm{A}$ exécuter des actes de fécondation, lorsque leur organisation les y sollicite ; $4^{\circ} \mathrm{A}$ rechercher des situations douces, des abris, etc. Les animaux intelligents, possèdent ce même penchant à fuir le mal-être et la douleur et le font par une variété d'actions bien supérieure à celle mis en œuvre par les animaux [seulement] sensibles. Le penchant à la conservation produit, notamment, chez ces animaux, une tendance vers le bien-être. Cette tendance vers le bien-être est d'un degré plus élevé que celle qui ne porte à fuir le mal-être que dans le cas seulement où on l'éprouve. La tendance vers le bien-être porte donc les animaux intelligents : $1^{\circ} \mathrm{A}$ se soustraire à la douleur et à tout ce qui les gêne ou les incommode ; $2^{\circ} \mathrm{A}$ rechercher les situations douces, avantageuses, les abris et le soleil dans les temps froids, l'ombre et le frais dans les temps chauds, etc., etc. $; 3^{\circ} \mathrm{A}$ satisfaire le besoin de se nourrir, quelquefois même avec voracité, soit par l'attrait qu'ils y trouvent, soit par l'inquiétude de manquer ensuite d'aliments ; $4^{\circ} \mathrm{A}$ se livrer aux actes de la fécondation, ou à en rechercher avec ardeur les occasions, lorsque leurs besoins provoqués les y sollicitent ; $5^{\circ} \mathrm{A}$ prendre du repos et sommeiller, lorsque leurs autres besoins sont satisfaits.".

Les temps modernes : l'animal confronté à des contraintes croissantes de la part des humains

Ces extraits de l'introduction du tome 1 des "Animaux sans vertèbres " rappellent étrangement les " libertés " du comité Brambell et de ce qui est préconisé actuellement 130 ans après leur publication. Sur le modèle des cinq libertés énoncées dans le premier amendement de la constitution des Etats-Unis 
d'Amérique, le FAWC (Farm Animal Welfare Council, 1979) reprend les recommandations du rapport Brambell de 1965 et énonce les libertés qui doivent être respectées pour placer les animaux en situation de bien-être :

"Freedom from hunger and thirst

Freedom from discomfort

Freedom from pain, injury and disease

Freedom to express normal behaviour

Freedom from fear and distress"

Dès les années 1980, le bien-être chez les animaux est considéré comme relevant de la « manière dont un animal perçoit et réagit à son environnement " et doit être élaboré en répondant à 2 questions : «Est-ce que les animaux sont en bonne santé ? » et «Est-ce que les animaux ont ce qu'ils veulent?» (Dawkins 1980). La question du bien-être animal, bien qu'étant revenue sur le devant de la scène récemment, a donc déjà été posée depuis longtemps et les outils pour y répondre ont été forgés scientifiquement depuis une quarantaine d'années. Si la controverse actuelle est vive, c'est que chacun s'approprie le comportement. Les conclusions des analyses scientifiques dépassent souvent le champ de la science et peuvent avoir des conséquences sur tous nos rapports avec les animaux, notamment en termes économiques. Il nous semble important de ne pas accepter la demande insistante de certains qui ne voudraient pas que ces questions soient abordées sous le prétexte que le simple fait de les poser risquerait de porter atteinte aux intérêts des filières. Il nous semble malsain d'ignorer les connaissances acquises au cours de ces décennies en relation avec le comportement voire de bloquer l'acquisition de connaissances dans ce domaine. Nous évoquerons dans ce texte les compétences des animaux dont la mise en évidence a connu un essor particulier au cours de ces dernières décennies (Figure 1). La mise en évidence expérimentalement de ces compétences rend irréfutable le fait que les animaux recherchent un bien-être et ressentent les situations où celui-ci est compromis. Dans cette perspective, nous aborderons trois notions : la cognition, la conscience et la douleur/ la souffrance (Figure 1). La douleur est définie comme la conscience de l'altération des tissus. La souffrance englobe la douleur mais également la peur et la détresse (Le Neindre et al., 2013).

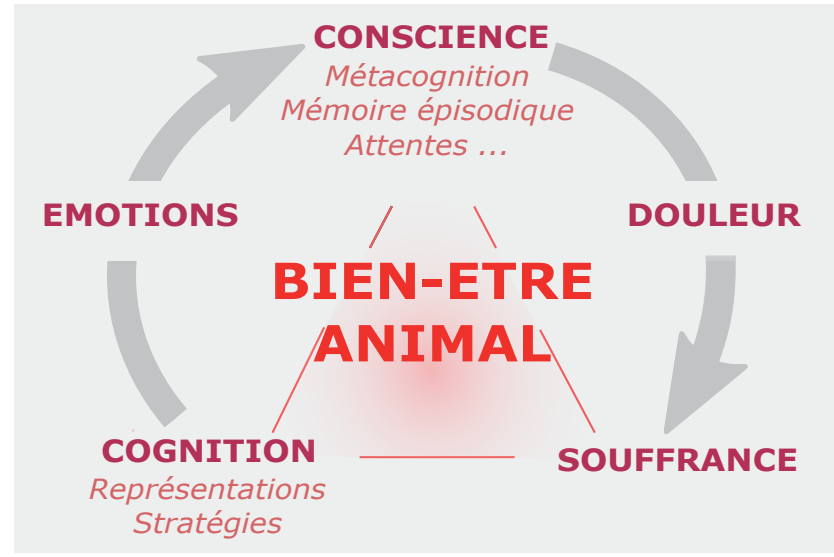

Figure 1. Les trois "piliers" du bien-être animal correspondants aux avancées récentes, au cours des 3 dernières décennies, de la recherche en éthologie, en psychologie comparée mais aussi en neurobiologie, imagerie et physiologie. Les flèches indiquent que la cognition est une composante essentielle de la conscience, elle-même essentielle pour la douleur.

\section{LES CONCEPTS-CLÉS DE L'ANALYSE DU BIEN-ÊTRE ANIMAL}

\section{La cognition chez les animaux}

Pendant très longtemps, et jusqu'à récemment, des auteurs ont avancé que les animaux obéissent à un faisceau de contraintes en y répondant de façon réflexe, "précablé ", ou par des processus préprogrammés issus de la sélection des animaux de l'espèce. La notion " d'instinct " était invoquée pour expliquer ces conduites (cf. Tinbergen 1951, Lorenz 1965a et b). Il a fallu un véritable travail de " déconstruction » pour requalifier les compétences des animaux. Il ressort de ces réflexions que, comme le proposaient déjà des contemporains de Descartes (Cureau de la Chambre 1647), il est légitime et parcimonieux d'avancer que certains animaux ont une autonomie de prise de décision qui n'est pas seulement le résultat de contraintes phylogénétiques. Dès 1912, C. Turner avait mis en avant la question de l'intentionnalité et des capacités cognitives (Samadi et al. 2020). Depuis plus de 50 ans la littérature éthologique, notamment sur les comportements des vertébrés supérieurs, a accumulé les évidences relatives aux prises de décisions des individus dans leur vie de relation et la plasticité de ces conduites, à travers notamment leurs capacités d'apprentissages (e.g. Campan \& Scapini 2002, Breed \& Moore 2012, Darmaillacq \& Lévy 2015). Au vu de toutes ces publications et de bien d'autres, il est clair que les vertébrés supérieurs, mammifères et oiseaux notamment, ne sont en aucun cas des robots mais des organismes capables d'apprentissages, donc doués de mémoire, leur permettant de prendre les décisions les plus adaptées face à la variété des situations auxquelles ils peuvent être confrontés.

Une synthèse complète est présentée dans l'ouvrage récent de Le Neindre \& Deputte (2020). Vauclair (1996) définit la cognition comme « l'ensemble des processus d'apprentissages et de traitement de l'information ; elle concerne la mise en œuvre de moyens appropriés pour atteindre un but donné ". La cognition repose sur la perception que l'individu a des éléments de son environnement biotique ou abiotique, sur la représentation qu'il en a et sur sa mémoire (Doré \& Mercier 1999). Elle permet aux individus de stocker les éléments perçus et ultérieurement d'effectuer des opérations sur les représentations de ces objets, comme dans le cas de reconnaissance individuelle et spécifique (Autier-Dérian et al. 2013). Elle porte sur la prise en compte par un individu des stimuli de l'environnement. Cette perception est modulée par l'état interne de l'animal. Des circuits neuronaux complexes et spécialisés, comme ceux relatifs à la vue, l'olfaction et l'ouie, transmettent des informations vers des parties spécifiques du système nerveux central. Ces signaux sont ensuite qualifiés par le cerveau en termes de valence et de pertinence. Chaque espèce possède un "monde vécu ", un "Umwelt » (von Uexküll 1934) dépendant de capacités perceptives qui leur sont propres. Les individus de certaines espèces perçoivent des ultra-sons (chiroptères, mais aussi chats et chiens), beaucoup d'autres n'ont pas cette aptitude. Certains, comme les oiseaux, perçoivent les ultra-violets. Enfin, d'autres, comme nos carnivores de compagnie ont des capacités olfactives particulièrement développées. Une notion centrale dans notre réflexion porte sur l'attente qui « est un processus mental généré par l'anticipation d'un évènement, auquel l'animal va se référer 
pour évaluer la valence de cet évènement, d'agréable à désagréable. " (Mormède et al, 2018). Cette notion de l'attente a fait l'objet de nombreux développements en psychologie comportementale. Nous proposons ici la description brève d'une expérience conduite sur des ovins. Des agnelles sont placées individuellement dans un enclos où elles ont l'habitude de recevoir de l'aliment dans une auge. Lors la distribution, l'apparition d'une planche colorée induit un sursaut de surprise. Si la planche est présentée après un son, les animaux anticipent cet évènement et n'ont plus de réaction la réaction de peur (Greiveldinger et al. 2007). Enfin afin de qualifier en partie le fait que les animaux sont capables de percevoir et de donner une valeur émotionnelle à leurs perceptions, certains auteurs ont voulu introduire, ou ré-introduire, le terme de sentience qui peut être défini de façon variable par les auteurs allant de la simple perception à la conscience telle que définie dans ce texte (voir Mormède et al, 2018). Ce terme polysémique ne nous parait soutenu par aucune définition opérationnelle. II ne nous parait ni essentiel ni utile, car il n'apporte rien de plus que les concepts déjà utilisés et qui, eux, ont conduit à un vaste corpus expérimental.

\section{La conscience des animaux}

Nous définissons la conscience des animaux comme «l'expérience subjective, ou phénoménale, que l'animal a de son environnement, de son propre corps et/ou de ses propres connaissances " (Le Neindre et al. 2017). Il s'agit donc de la réflexivité de l'animal sur lui-même et sur sa place dans son environnement. Il est extrêmement important de souligner qu'il est nécessaire de distinguer cette définition de celle prise pour la conscience morale qui décrit comment l'homme distingue le bien du mal, l'acceptable de l'inacceptable. Cette dernière acception n'est pas illégitime mais elle est du ressort de la morale et pas de celui de la science. Le concept de conscience est longtemps resté un sujet tabou pour les éthologistes objectivistes. Griffin (1976) a cependant justement considéré qu'il était plus parcimonieux d'utiliser ce concept pour expliquer la complexité des comportements des animaux, leur flexibilité et leur plasticité, que d'invoquer des ensembles complexes de relations "stimulus-réponse ". Ce qu'il a tout de même appelé avec prudence « l'awareness " - que l'on peut tenter de traduire par " prise de conscience » est devenu un champ expérimental pour les éthologistes cognitivistes qui se rapprochaient alors des psychologues comparatistes (cf. Morgan, 1894). Pendant très longtemps, il a été dit que cette conscience réflexive était le propre de l'homme. Des déclinaisons sur différents aspects ont été publiées pour renforcer la spécificité des compétences de l'homme en termes de conscience. Il a été dit que l'humain a conscience en particulier de lui, de ses connaissances, de celles des autres, de son passé et de son futur. Des résultats scientifiques appuient ces assertions (par exemple Searle, 1995). Pour étayer ces conclusions, les chercheurs s'appuyaient essentiellement sur le discours des humains. Cependant, il est apparu que cette contrainte n'était pas falsifiable lorsque les humains ne pouvaient pas parler, ce qui est le cas pour certains malades mentaux, pour des personnes dans des états non complétement conscients, comme les comateux, et pour les nouveau-nés et bien sûr pour les animaux. Suite à la proposition de Griffin (1976), des dispositifs expérimentaux ont donc été mis en place pour s'affranchir de la nécessité d'un rapport verbal (de
Waal, 2016). Le niveau et le contenu de la conscience sont distingués. Le niveau peut varier de la pleine conscience à la pleine inconscience. Ces différences sont importantes non seulement d'un point de vue théorique mais également d'un point de vue pratique. Ainsi, le niveau de conscience conduit à caractériser le coma, le sommeil, et l'éveil aussi bien chez les humains que chez les animaux. Les chirurgiens vétérinaires anesthésient les animaux pour réduire le niveau de conscience et pas seulement pour réduire les risques d'accident. A l'abattoir, les vétérinaires inspecteurs sont garants du fait que les animaux sont effectivement inconscients avant d'être saignés. Si l'abattage sans étourdissement pour des raisons religieuses interpelle certains, c'est que cela revient à égorger des animaux alors qu'ils sont conscients. Mais nous souhaitons surtout poser la question du contenu de la conscience, à quoi pensent-ils ? et pas seulement pensent-ils ? Nous évoquerons notamment les émotions, le voyage dans le temps, c'est-à-dire la conscience du passé et du futur, et la métacognition c'est-à-dire la conscience de sa connaissance. La conscience suppose une dimension émotionnelle modulant la réaction de l'animal. Encore une fois, nous voulons mettre en exergue le fait que l'animal ne réagit pas mécaniquement à son environnement. Il réagit en fonction de la valence de cet environnement, de l'intérêt qu'il y porte, qui est fonction de sa motivation et de son passé. Boissy et al. (2007) ont montré que les animaux réagissaient à un évènement nouveau par une réaction émotionnelle qui est fonction de plusieurs éléments et en particulier de son anticipation et de son passé. Ainsi, des ovins qui ont eu ou non des expériences perturbantes vont réagir de façons différentes. Contrairement à ce qui avait été postulé auparavant, les animaux possèdent, comme les humains, une mémoire épisodique qui leur permet de savoir quel événement ils ont vécu, où et quand. Cette compétence a été démontrée sur de multiples espèces. Kouwenberg (2009) rapporte une expérimentation conduite chez des porcs. Elle s'appuie sur le fait que les porcs choisissent souvent de passer plus de temps à explorer des objets nouveaux que des objets familiers. Les porcs sont familiarisés avec deux objets différents placés d'un côté d'une arène ou d'un autre, sur un sol qui est soit gris, soit blanc. Le même objet leur est ensuite présenté du côté et sur le sol de couleur où ils ne l'ont pas vu auparavant. Ils choisissent d'explorer préférentiellement l'objet dans l'environnement le moins familier. Ils montrent ainsi leur capacité à différencier de quels objets ils se souviennent, où et quand et donc leur capacité à avoir une mémoire épisodique. Cette capacité à se situer dans le temps est valide également pour planifier des évènements futurs. Soley \& Alvarado-Diaz (2011) décrivent les activités de martres à tête grise, Eira barbara, qui les conduisent à conclure à cette compétence. Enfin, il a été montré que les animaux ont des compétences métacognitives, c'est-à-dire qu'ils sont capables d'agir en sachant qu'ils connaissent ou pas la bonne réponse. Par exemple, Foote $\&$ Crystal (2007) mettent à disposition de rats trois pédales. L'appui sur une des deux premières pédales permet aux rats d'obtenir une récompense s'ils répondent correctement à la question. Ils peuvent avoir à discriminer entre deux sons de durées différentes ou entre différents mélanges d'odeurs. Si le rat appuie sur la pédale correspondant au son, ou à l'odeur, choisi par l'expérimentateur, il reçoit une forte récompense, sinon il n'a rien. S'il appuie sur la troisième pédale, il reçoit une petite récompense dans 
tous les cas. Par la suite, les caractéristiques des stimuli correspondant aux deux premières pédales deviennent de moins en moins faciles à distinguer. Lorsque la discrimination devient quasi impossible, on observe que les rats appuient de plus en plus souvent sur la troisième pédale. Les auteurs concluent que les rats montrent qu'ils savent ou qu'ils ne savent pas répondre correctement à la question de discrimination et donc qu'ils ont des capacités métacognitives. Ces quelques exemples illustrent le fait que nous sommes capables désormais de démontrer scientifiquement l'existence de ces compétences. Ils permettent de mieux comprendre comment les animaux évaluent les situations en termes de bien-être ou de mal-être.

\section{Douleurs des animaux}

Le concept de douleur chez les humains a fait l'objet d'évolutions significatives dans les trente dernières années. Il y a quarante ans, la douleur était définie comme une sensation traduisant un traumatisme, une agression tissulaire ou une inflammation. Cependant, cette définition s'est avérée insuffisante pour décrire différents types de douleurs comme par exemples les douleurs aigüe, inflammatoire, chronique, périphérique ou viscérale. Désormais, l'IASP (International Association for the Study of Pain) définit la douleur comme « une expérience sensorielle et émotionnelle désagréable, associée à une lésion tissulaire, réelle ou potentielle ou décrite en termes évoquant une telle lésion ". L'évaluation de la douleur introduit la notion de la subjectivité de l'individu dans son ressenti. De plus, cette définition permet de considérer la douleur chez des humains non verbaux comme les nouveau-nés, les malades mentaux et les personnes non pleinement conscientes. L'évaluation n'est plus limitée à l'autoévaluation mais peut être une hétéroévaluation. Cette introduction de l'hétéroévaluation chez les humains permet une convergence avec l'évaluation de la douleur chez les animaux qui a été étudiée dans le cadre d'une expertise collective de l'INRA (2013). Molony \& Kent (1997) définissent la douleur comme « une expérience sensorielle et émotionnelle aversive représentée par la conscience que l'animal a de la rupture ou de la menace de rupture de l'intégrité de ses tissus ». Ce ne sont donc pas les modifications des tissus qui provoquent la douleur mais la conscience que l'animal a de ces changements. Dans ces conditions, la conscience devient un passage obligé et les considérations concernant ses niveaux et ses contenus prennent une grande importance. Le mandat de l'expertise sur les douleurs animales en élevage stipulait " comment traite-t-on la douleur aujourd'hui en élevage et quelles sont les limites à sa prise en compte " (Le Neindre et al. 2013, p.2). A partir de l'analyse des connaissances biologiques et techniques qui ont été développées dans la première partie de cette expertise collective, il a été possible de proposer des pistes pour réduire la douleur des animaux. Afin de structurer ces pistes possibles de progrès, l'expertise a proposé la démarche des trois S : Supprimer, Substituer et Soulager. Elle a identifié des pratiques qui pourraient être supprimées car le bénéfice qu'elles apportent ne contrebalance pas le coût en termes de douleurs. C'est le cas par exemple de la coupe des queues des vaches laitières et des chevaux de trait. Certaines techniques pourraient être substituées par d'autres qui induisent moins de douleurs. Ainsi, la castration à vif des animaux peut être remplacée par une immunocastration. Enfin, lorsque des actes moins douloureux ne sont pas disponibles il convient de soulager la douleur induite en utilisant des traitements pharmacologiques. Cette stratégie peut être mise en œuvre par exemple, dans le cas de mutilations lors de l'écornage, ou de la castration des chevaux, bovins et ovins.

\section{DISCUSSION}

Ces réflexions sur les capacités cognitives, la conscience et la douleur des animaux sont essentielles pour clarifier les idées que nous devons mettre en avant lorsque la question du bien-être animal est abordée. Mais elles sont surtout importantes d'un point de vue pratique. Si des mesures doivent être prises à un niveau réglementaire, il est nécessaire de rappeler le caractère individuel du ressenti des animaux. La bientraitance est nécessaire pour le maintien du bien-être animal mais pas suffisante car elle se traduit par des mesures applicables à des cohortes ou des groupes d'individus et non pas à un individu donné. Etant donné cette nature individuelle, il devient nécessaire de vérifier pour chaque individu la pertinence des mesures de bientraitance.

Les résultats scientifiques montrent que les animaux maîtrisent leur passé et planifient leur futur et qu'ils ont conscience de leurs connaissances. Ils ont donc des attentes dont la principale est de trouver un niveau de bien-être qui les satisfasse individuellement en évitant les situations qui le compromettent. Ils montrent aussi que les animaux ne sont pas les "animaux-machines" incapables de souffrir et donc qui ne peuvent être les objets de toute contrainte (Le Neindre, 2000).

Le bien-être animal ne peut pas être décrété par ceux qui parlent au nom des animaux, mais qui ne les observent pas. Le bien-être animal est une thématique scientifique dont les applications ont des retombées importantes en matière de respect des animaux et de la relation homme-animal. Le bien-être animal peut se passer de "l'amour " à l'égard des animaux mais impose une observation attentive de leurs réactions dans tous les moments de leur vie quotidienne, quelle que soit leur espérance de vie.

Nous avons voulu montrer tout cela en présentant ce que la recherche nous enseigne sur ce que des animaux peuvent avoir dans leur pensée, et ce que la recherche nous permet de mettre en place par une observation structurée, opérationnelle et donc efficace pour évaluer les états individuels de bien-être. Donc, le bien-être n'est pas un phantasme d'amoureux de la nature, ni le reflet d'une sensiblerie, justifiée ou ressentie, à l'égard des animaux, notamment ceux qui sont soumis par l'homme, Homo sapiens, à toute forme de restrictions et de contraintes. Ce n'est pas un concept creux, une coquille vide de sens même si le mot est employé souvent à tort et à travers, comme un tic langagier. Ce n'est pas non plus un mot " auberge espagnole " où chacun s'arroge le droit de le définir comme il veut pour défendre ses intérêts. Il n'est pas question ici de l'intérêt de l'humain mais de celui des animaux qu'il contraint et du respect légitime qu'il leur doit eu égard aux services divers et variés qu'ils lui rendent. Le bien-être animal est un concept d'éthologistes et de psychologues comparatistes. Son appréciation passe nécessairement par 
l'observation des animaux en tant qu'individus d'une espèce donnée.

L'analyse qui en découle permet aux différents acteurs de terrain de mettre en place les nécessaires mesures de bientraitance. Il y a en fait deux approches de la problématique de l'interaction animal/milieu, celle du bien-être animal, qui conduisent à une réflexion éthique :

- soit les individus sont modifiés génétiquement par une sélection artificielle imposée par l'homme pour que le "produit " soit ajusté aux contraintes que l'on a décidé de leur imposer.

- soit les contraintes sont ajustées afin que l'animal puisse s'y adapter et donc trouve dans ses ressources biologiques les solutions aux problèmes que nous lui posons (Figure 2).

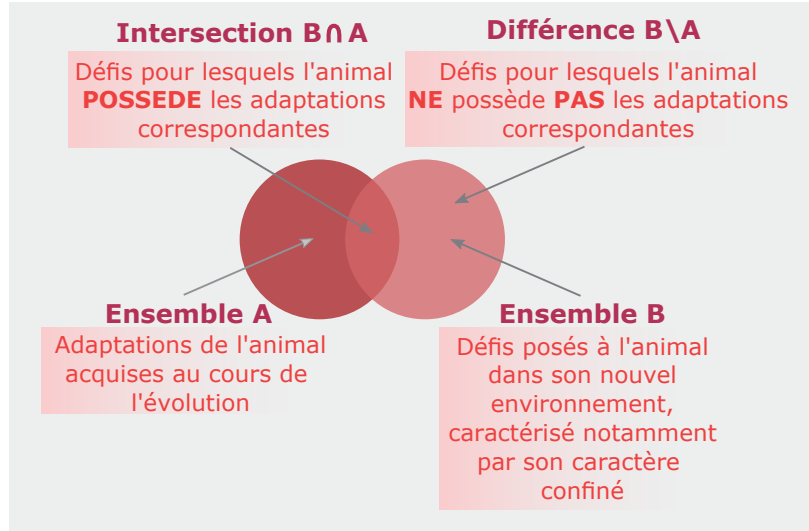

Figure 2. Diagramme de Venn représentant la manière avec laquelle les animaux notamment domestiques, peuvent répondre aux contraintes qui leur sont imposées par les pratiques d'élevage (d'après Fraser et al. 1997). La réflexion doit porter sur soit une réduction de la quantité des contraintes - ensemble B ; soit l'adaptation de ces contraintes aux adaptations des animaux et à leurs compétences. Ces deux actions conduiront à une augmentation de la taille de l'intersection $B \cap A$, en rouge vif, synonyme de maintien $d u$ bien-être pour les animaux et corrélativement à une réduction de celle de la différence $B \backslash A$, en rouge pâle.

Ce modèle postule deux ensembles distincts, d'une part (A) celui des adaptations acquises au cours de l'évolution pour les individus d'une espèce donnée et d'autre part (B) celui des défis posés aux animaux dans les conditions de confinement imposées par les humains. L'intersection de ces deux ensembles $(\mathrm{B} \cap \mathrm{A})$ inclut les défis pour lesquels les animaux trouvent des solutions dans leurs compétences spécifiques. Il reste une partie du $2^{\text {ème }}$ ensemble $(B \backslash A)$ qui représente les défis pour lesquels les animaux n'ont pas d'adaptation correspondante et ne peuvent les acquérir du fait du temps de vie raccourcie imposée par les humains. Selon ce modèle, les mesures de bientraitance ou les contraintes que les humains décident d'imposer doivent tendre à ce que l'intersection de ces 2 ensembles soit la plus importante possible (Fig. 2). Dans ce cas, l'impératif n'est pas génétique mais comportemental et nécessite une évaluation scientifique de ce que les individus d'une espèce donnée font, ressentent et perçoivent de situations contraignantes dans lesquelles les humains les ont placés pour leur propre satisfaction.

Transformer les animaux pour qu'ils s'adaptent aux contraintes croissantes (intervenir sur l'ensemble A de la figure 2).

Pour les tenants de la première approche prônant une modifica- tion génétique dans son acception extrême " modifier l'animal ", celui-ci devient "désanimalisé ». Il est progressivement rabaissé au rang d'objet manufacturé. Ses adaptations, ses compétences biologiques sont gommées, évacuées, deviennent obsolètes à l'exception de celles qui servent l'homme. Ipso facto la question du bien-être animal est évacuée, on ne parle plus que de technologies génétiques. Le comportement - la vie de relation- des individus appartenant à une espèce donnée, ne rentre plus dans la réflexion.

Ajuster les contraintes aux potentialités des animaux (intervenir sur l'ensemble B de la figure 2)

Pour les tenants de la 2ème approche - celle de l'adaptation des contraintes-, il est question de permettre à l'animal d'utiliser les spécificités biologiques et les traits d'histoire de vie qui ont fait que l'homme, au cours de sa propre évolution, il y a une dizaine de millénaires, ait choisi cette espèce pour telle ou telle utilisation à son profit (alimentation, transport, sport, compagnie). Driscoll \& Bateson (1998) ont proposé un modèle de décision éthique relatif à la recherche scientifique expérimentale utilisant les animaux. Ils l'ont bâti sur trois variables : la qualité de la recherche, l'assurance des bénéfices des résultats de cette recherche pour l'homme, et la souffrance animale. Les zones du modèle où un projet de recherche est acceptable sont celles où la souffrance infligée aux animaux est négligeable, la qualité de la recherche élevée et le bénéfice certain.

Nous proposons d'adapter ce modèle dans le cadre de l'élevage. Le bien-être pourrait être substituer à la souffrance, la qualité du produit à celle de la recherche et le prix de ce produit à la certitude du bénéfice. Un élevage éthique serait alors celui où le produit serait de qualité en respectant un niveau élevé de bien-être pour les animaux et où le prix de ce produit serait peu élevé. A l'heure actuelle les zones de ce modèle les plus observées sont 1 - celle où le bien-être est minimal - même s'il est conforme à la réglementation -, le produit de moindre qualité et un prix bas, 2-à l'opposé, celle où le respect du bien-être conduit à un produit de qualité mais à un prix élevé. Le compromis économique à réaliser devra nécessairement se faire dans la partie du modèle où le bien-être animal est élevé s'il est, comme on l'entend et le souhaite, une préoccupation essentielle et non une coquille vide. Adapter le niveau de contraintes aux adaptations des animaux et à leur capacité d'y répondre relève de l'expression du respect que l'on peut - ou doit-avoir pour l'animal.

\section{CONCLUSION}

Ce respect devrait être aussi la marque de notre regard vers le règne animal - sans " roi ", comme le souligne si bien Budiansky (2002) : "Toutes les créatures ont trouvé des voies singulières pour exister et survivre contre toute probabilité. Et c'est cela que nous devons respecter et chérir. ». Il est important que le bien-être des animaux soit devenu un sujet sociétal, Mais il est encore plus important de veiller à ce qu'il ne soit pas détourné de sa réalité biologique et que ceux qui font évoluer les outils d'évaluation soient régulièrement interrogés et leur avis régulièrement pris en compte, dans une concertation évidemment multipartite. Comme le souligne Weiskopff (1994) : "L'existence humaine dépend de la compassion et de la connaissance. La connaissance sans compassion est inhumaine, la compassion sans connaissance est inefficace." 


\section{REMERCIEMENTS}

Nous remercions en premier lieu Jean-Luc Angot, président de cette académie, d'avoir mis à l'ordre du jour cette question qui a fait l'objet de vifs débats dans la communauté scientifique mais qui nous parait désormais suffisamment mâture. Nos sincères remerciements vont aussi aux relecteurs qui ont permis une amélioration significative de ce texte

\section{BIBLIOGRAPHIE}

- Autier-Dérian D, Deputte BL, Chalvet-Monfray K, Coulon M, Mounier L. Visual discrimination of species in dogs (Canis familiaris). Anim Cogn. 2013 ; 16: 637-651.

- Bernard C. Leçons sur la physiologie et la pathologie du système nerveux. Cours de médecine du Collège de France. J.B. Baillière \& fils, Paris. Tome 1; 1858.

- Boissy A, Manteuffel G, Jensen MB, Moe RO, Spruijt B, Keeling L, et al.. Assessment of positive emotions in animals to improve their welfare. Physiol and Behav. 2012; 92: 375-397.

- Breed MD \& Moore J. Animal Behavior, Burlington, MA, Academic Press; 2012.

- Budiansky S. If A Lion Could Talk: Animal Intelligence and the Evolution of Consciousness. New York Free Press; 1998.

- Campan R \& Scapini F. 2002. Le comportement animal : approche systémique du comportement. De Boeck, Paris ; 1998.

- Cureau de la Chambre M. Traité de la connoissance des animaux, où tout ce qui a esté dict pour et contre le raisonnement des bestes est examiné, Pierre Rocolet, Paris ; 1647.

- Darmaillacq AS \& Lévy F editors. Ethologie animale : une approche biologique du comportement. Louvain la neuve, Belgique, De Boeck; 2015.

- Darwin C. On the Origin of Species by Means of Natural Selection, or the Preservation of Favoured Races in the Struggle for Life, J. Murray, London; 1859.

- Dawkins MS. Animal Suffering: The Science of Animal Welfare. Chapman and Hall, London ; 1980.

- Descartes R. EEuvres de Descartes. Paris : J. Vrin ; (1596-1650)1996.

- Doré FY \& Mercier P. Les Fondements de l'apprentissage et de la cognition. Gaetan Morin Quebec; 1999.

- Driscoll JW \& Bateson P. Animals in behavioural research. Anim. Behav., 1988; 36: 1569-1574.

- FAWC, 1979. Farm Animal Welfare Council. Press statement. Disponible à http://webarchive.nationalarchives. gov.uk/20121007104210/http:/www. fawc.org.uk/pdf/fivefreedoms 1979.pdf (consulté le 2.12.2020).

- Foote AL \& Crystal JD. Metacognition in the rat. Current Biology. 2007; 17: 551-555.

- Fraser D, Weary DM, Pajor EA, Milligan BN. A scientific conception of animal welfare that reflects ethical concerns. Anim. welfare. 1997; 6: 187-205.

- Greiveldinger L, Veissier I, Boissy A. Predictability and expectations influence emotional responses in lambs. Anim. Welfare. 2007; 16: 170.

- Griffin D. The question of animal Awareness. The Rockefeller University Press, New York; 1976.

- Inglehart $\mathrm{R} \& \&$ Klingemann HD. Genes, democracy and happiness In: Subjective well-being across cultures. Diener E, Suh EM, editors. Cambridge MA, MIT Press; 2000, pp 165-183.

- Kouwenberg AL, Walsh CJ, Morgan BE, Martin GM. Episodic-like memory in crossbred Yucatan minipigs (Sus scrofa). Appl Anim Behav Sci. 2009; $117: 165-172$.

- Lamarck de JBPA. Histoire naturelle des animaux sans vertèbres. Tome 1 , Paris, J.B. Baillière ; 1835.

- Le Neindre P. Positions éthiques, structures de réflexion et de décision en matière de bien-être animal. Bull. Acad. Vét de France. 2000 ; 153: 263-271.

- Le Neindre P, Baldin D, Desmoulin S, Guatteo R, Guémené D, Guichet JL et al. Douleurs animales en élevage, Ed Quae ; 2013.

- Le Neindre P, Bernard E, Boissy A, Boivin X, Calandreau L, Delon N, et al. Animal Consciousness. J. EFSA Journal, Ed. Wiley ; 2017.

- Le Neindre P \& Deputte BL. Vivre avec les animaux, mieux les comprendre. Editions Quae ; 2020.

- Le Petit Robert, édition 1977.
- Lorenz K. Essais sur le comportement animal et humain. Paris, Seuil; 1965a.

- Lorenz K. Evolution and Modification of Behavior, Chicago, The University of Chicago Press; 1965b.

- Milhaud C. Vers le bien-être des animaux de laboratoire par la bientraitance. Sci Tech Anim Lab. 2006 ; 2 :135-140.

- Milhaud C., 2007. Rapport sur l'utilisation du néologisme « bientraitance » à propos de la protection des animaux, académie vétérinaire de France, 29pp.

- Morgan CL. Introduction to comparative psychology. London, W. Scott; 1894.

- Mormède P, Boisseau-Sowinski L, Chiron J, Eddison J, Guichet JL, Le Neindre P. Bien-être animal : contexte, définition, évaluation. Prod anim. 2018 ; 31 : 145-162.

- Samadi H, Dona G, Chittka L C. Turner. Pioneer in animal cognition. Science. 2020; 370: 530-531.

- Searle JR. The mystery of consciousness. New York Review of books, 1995; 42: 60-66.

- Soley FG \& Alvarado-Diaz I. Prospective thinking in a mustelid? Eira barbara (Carnivora) cache unripe fruits to consume them once ripened. Naturwissenschaften. 2011; 98: 693-698.

- Tinbergen N. The study of instinct. Oxford, Clarendon Press; 1951.

- Tinbergen N. On aims and methods of Ethology. Z für Tierpsychol. 1963; 20: 410-433.

- Uexküll von J. Mondes animaux et monde humain suivi de La théorie de la signification, (trad. fr. éd. Denoël, Paris, 1965) ; 1934.

- Vauclair J. La cognition animale. Paris. P.U.F. Coll. "Que sais-je?" ; 1996.

- Waal de F. Are we smart enough to know how smart animals are? Granta; 2016.

- Waal de F. Fish, mirrors, and a gradualist perspective on self-awareness. PLOS Biol. 2019; 17: e3000112

- Weiskopff V. Essay: Endangered support of basic science. Sci Amer. $1994 ; 270$ : 96. 